\title{
Clinical evaluation of the Allergan Humphrey 500 autorefractor and the Nidek AR-1000 autorefractor
}

\author{
Bettina Kinge, Anna Midelfart, Geir Jacobsen
}

\begin{abstract}
Aims/Background-The intentions of this study were to estimate agreement between two different autorefractors and standard subjective refraction techniques and to evaluate the clinical implications of relying on the autorefractor measurements.
\end{abstract}

Methods-Subjective refraction was carried out on 448 cycloplegic eyes and compared with cycloplegic readings with the Allergan Humphrey 500 autorefractor (448 eyes) and the Nidek AR-1000 autorefractor (160 eyes). Each refraction was followed by clinical visual acuity measurement. The study population comprised 224 healthy students, 107 men and 117 women, with a mean age of 20.6 (SD 1.1) years.

Results-Both the Nidek and Humphrey autorefractors measured more negative or less positive refractive values compared with subjective refraction and these biases were statistically significant (Humphrey right eye $-0.23 D, p=0.0001$, left eye $-0.20 \mathrm{D}, \mathbf{p}=\mathbf{0 . 0 0 0 1}$ ), (Nidek right eye $-0.13 \mathrm{D}, \mathrm{p}=0.0001$, left eye $-0.11 \mathrm{D}$, $p=0.0002)$. Comparing the results of autorefraction with subjective refraction, the Nidek was better than the Humphrey autorefractor in several ways: a smaller mean difference, better agreement between spherical equivalent values, narrower limits of agreements, and better visual acuity obtained with the autorefraction. On the other hand, the Humphrey autorefractor agreed better with subjective refraction concerning cylinder axis.

Conclusion-The results show that both autorefractors represent a valuable complement to subjective refraction, but cannot be used as a replacement.

(Br f Ophthalmol 1996; 80: 35-39)

The University of Trondheim, Faculty of Medicine, Trondheim, Norway

Department of Ophthalmology B Kinge

A Midelfart

Department of Community Medicine and General Practice G Jacobsen Correspondence to: Dr Bettina Kinge, Nedre
Silkestrå 18, N-0375 Oslo, Norway.

Accepted for publication 14 August 1995

Automatic objective refractors, which estimate the refractive error without requiring any operator or patient judgment, have been available since the early 1970s. These instruments are easy to operate, are much quicker than manual refraction, and are appreciated by the patients. ${ }^{1}$ Therefore, there is an increased acceptance for them in clinical settings.

Many of the previous comparative studies made with automatic refractors have been done without cycloplegia and have focused mainly on repeatability, accuracy, and ease of use. Comparing autorefractors of different manufacturers, Wesemann and Rassow concluded that the differences in accuracy between these had become very small. ${ }^{2}$ Nevertheless, for some instruments, myopia due to accommodation and inadequate autofogging mechanisms have been revealed. ${ }^{2-4}$ Comparing clinical refraction with autorefraction on cycloplegic eyes, various degrees of agreement between these refraction methods were reported..$^{5-7}$

Previous studies have based their conclusions largely on comparison of mean values and on correlation coefficients, or in some cases, on the results of regression analysis. In this study, analysis of limit of agreement was performed in agreement with recommendations given by Bland and Altman. ${ }^{8}$

This study was initiated to evaluate two different autorefractors often used in clinical practice. It was carried out on cycloplegic eyes to control latent hyperopia or pseudomyopia.

The aims of our study were to (1) estimate agreement between refractive error measured with either Humphrey or Nidek autorefractors and standard subjective refraction techniques; and (2) evaluate the clinical implications of relying on the autorefractor measurements.

\section{Materials and methods}

A group of 224 first year students at the Norwegian Institute of Technology (NTH) took part in this study. They were selected at random and were a representative sample of the Norwegian engineering students at the NTH. ${ }^{9}$ Of these, $107(47.8 \%)$ were males and $117(52 \cdot 2 \%)$ were females, with a mean age of

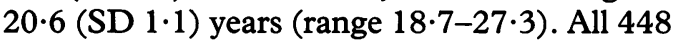
eyes were included in the study because no subject fulfilled exclusion criteria of aphakia, pseudophakia, diabetes mellitus, other eye disease, or eye injury. Two persons had amblyopia (visual acuity below 0.5 in one eye each due to hyperopia (one) and anisometropia (one)).

The eye examinations took place at the

Department of Ophthalmology, Faculty of Medicine at the University of Trondheim, Norway and all the measurements were carried out by the first author using the same procedure and equipment. After slit-lamp examination of the anterior segment of the eye, one drop of cyclopentolate $1 \%$ was administered to each eye and repeated after 5 minutes. The examination was continued after 30 minutes. The power of corrective lenses, if any, was measured in a lensometer (Allergan, Humphrey lens analyser).

All 448 eyes were analysed using an 


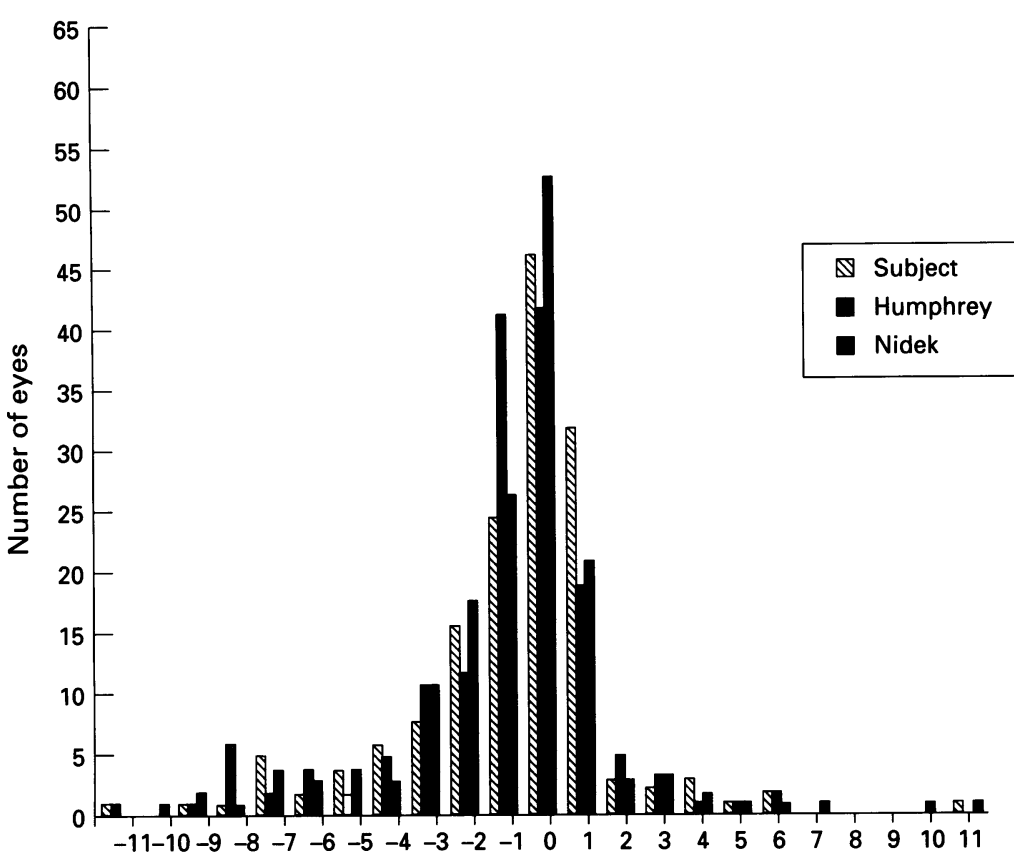

Refractive equivalent (D)

Figure 1 Distribution of refractive equivalent values ( $n=160$ ).

Allergan Humphrey 500 autorefractor. A 'standardised' value was displayed after a series of measurements had been taken in the measurement cycle. ${ }^{4}$ Subsequently, all students were subjectively refracted using the subjective refraction method. In addition, 80 students were selected at random from the study population and their eyes $(n=160$ eyes) were analysed using a Nidek AR-1000 autorefractor, obtaining three measurements and from which the average value was calculated. To summarise, the refractive examination in cycloplegia was carried out using the following procedure commencing with right eye: (1) autorefraction by Humphrey; (2) autorefraction by Nidek if selected; (3) clinical measurement of visual acuity according to the results of autorefraction by Humphrey; (4) clinical measurement of visual acuity according to the results of autorefraction by Nidek, if any; (5) standard subjective refraction; (6) clinical measurement of visual acuity according to the subjective refraction.

The study has been approved by the regional ethics committee.

Table 1 Agreement between either the Humphrey 500 autorefractor (HUM) or the Nidek $A R-1000$ autorefractor (NID) and subjective refraction (SUB) in right and left eyes. The $95 \%$ confidence interval (CI) of the mean differences and of the limits of agreement have been calculated $^{8}$

\begin{tabular}{|c|c|c|c|c|}
\hline & \multicolumn{2}{|c|}{ Right eyes (D) } & \multicolumn{2}{|c|}{ Left eyes (D) } \\
\hline & & $95 \% C I$ & & $95 \% C I$ \\
\hline \multicolumn{5}{|l|}{ HUM-SUB: } \\
\hline Number of eyes & 224 & & 224 & \\
\hline Mean difference & $-0 \cdot 23$ & $-0 \cdot 29$ to $-0 \cdot 17$ & $-0 \cdot 20$ & -0.26 to -0.14 \\
\hline Lower limit of agreement & $-1 \cdot 18$ & -1.29 to -1.07 & $-1 \cdot 12$ & -1.23 to -1.02 \\
\hline Upper limit of agreement & $0 \cdot 71$ & 0.60 to 0.82 & 0.72 & 0.62 to 0.83 \\
\hline \multicolumn{5}{|l|}{ NID-SUB: } \\
\hline Number of eyes & 80 & & 80 & \\
\hline Mean difference & $-0 \cdot 13$ & -0.19 to -0.07 & $-0 \cdot 11$ & -0.17 to -0.06 \\
\hline Lower limit of agreement & -0.68 & -0.79 to -0.58 & -0.62 & -0.72 to -0.52 \\
\hline Upper limit of agreement & 0.41 & 0.31 to 0.52 & $0 \cdot 40$ & 0.30 to 0.50 \\
\hline
\end{tabular}

PRESENTATION OF THE RESULTS

The refractive values were converted into spherical equivalents for some analysis (=sphere value +0.5 of the cylinder value).

The differences between the refraction values made by the Humphrey autorefractor (HUM), the Nidek autorefractor (NID), and subjective refraction (SUB) were calculated as follows:

Difference between (HUM and SUB)= HUM-SUB

Difference between (NID and SUB) $=$ NID-SUB

A Shapiro-Wilk test was used to test for normality of the differences in spherical equivalent values between either Humphrey or Nidek autorefraction and subjective refraction. ${ }^{10}$ The mean differences with $95 \%$ confidence intervals (CI) and 95\% limits of agreement (mean difference plus or minus 2 standard deviations) were calculated, according to Bland and Altman. ${ }^{8}$ The precision of estimated limits of agreement is expressed by the $95 \% \mathrm{CI}$ of these limits.

Student's, McNemar, and Kruskal-Wallis tests were employed to determine the statistical differences. ${ }^{10}$ All errors are expressed as standard deviations (SD) and the level of statistical significance was $5 \%$.

The data were stored on a personal computer and statistical analysis was performed using the Statistical Analysing System (SAS). ${ }^{10}$

\section{Results}

The distribution of the spherical equivalent values among the 160 eyes refracted both subjectively by the Humphrey autorefractor and the Nidek autorefractor is shown in Figure 1. The figure illustrates the asymmetrical distribution of refractive errors with a tail in the myopic direction. Determined with standard subjective refraction, the range of refractive errors $(n=160)$ was 11.0 to $-10.5 \mathrm{D}$, and the mean refractive value was -0.7 (SD 2.5) $\mathrm{D}$ (right eye), and -0.7 (SD 2.9) D (left eye).

\section{AGREEMENT BETWEEN REFRACTION}

DETERMINED BY THE HUMPHREY

AUTOREFRACTOR AND SUBJECTIVE REFRACTION As shown in Table 1 , the mean differences in spherical equivalent between the refraction employing the Humphrey autorefractor and the subjective refraction were $-0.23 \mathrm{D}$ (right eye) and $-0.20 \mathrm{D}$ (left eye). These values were significantly different for both eyes (right eye $\mathrm{p}=0.0001$, left eye $\mathrm{p}=0.0001$ ). In 220 of 448 $(49 \cdot 1 \%)$ eyes the spherical equivalent values measured by the autorefractor agreed with the subjective refraction values within plus or minus $0.25 \mathrm{D}$, and in $328(73.2 \%)$ eyes agreed within plus or minus $0.50 \mathrm{D}$. Regarding the sphere component, values determined in 253 eyes $(56.5 \%)$ by the autorefractor agreed with the subjective refraction within plus or minus $0.25 \mathrm{D}$, and in 354 eyes $(79.0 \%)$ within plus or minus 0.50 D. Furthermore, in 377 eyes $(84 \cdot 2 \%)$ the cylinder component determined by these two methods was within plus or minus 


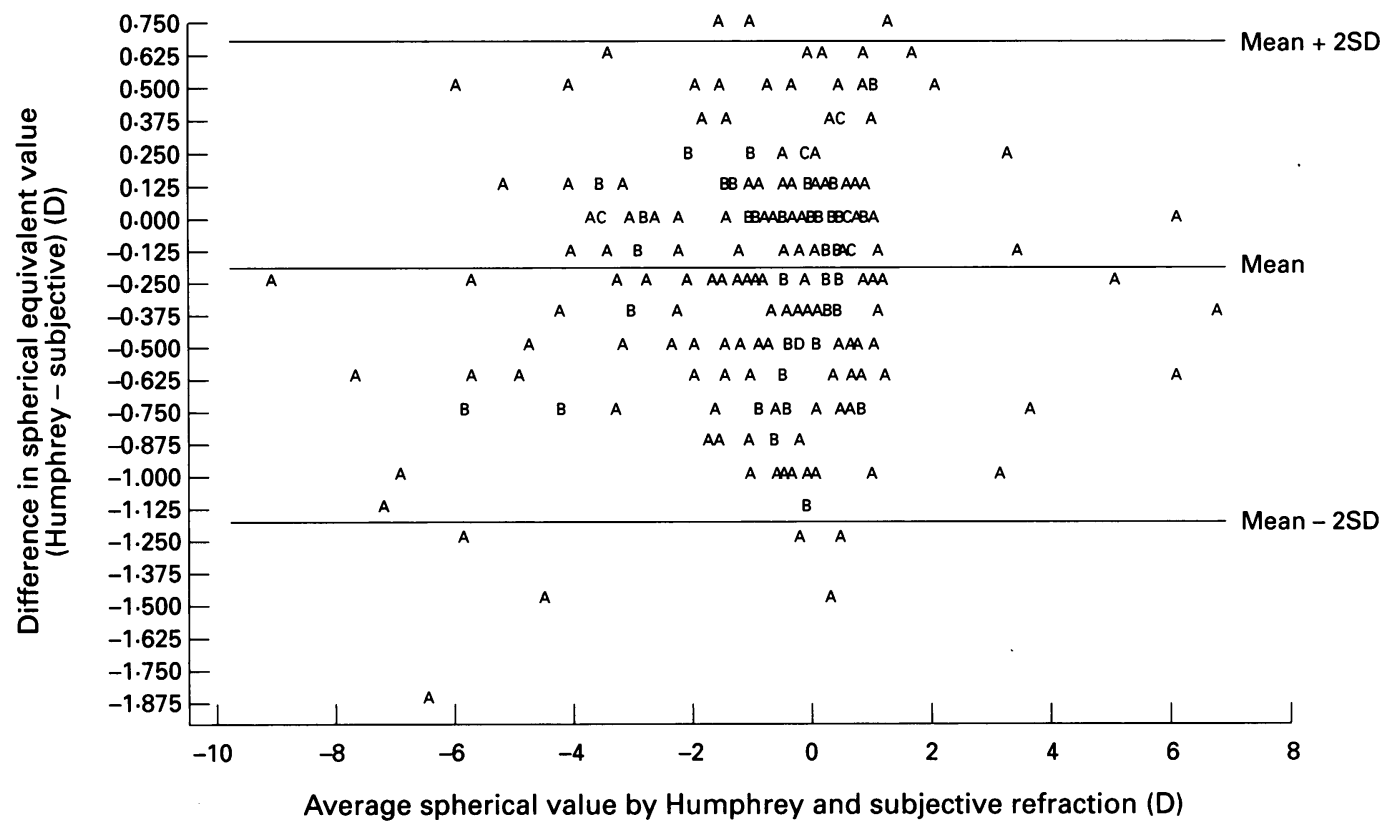

Figure 2 Bland and Altman plot of the average spherical equivalent value by Humphrey and subjective refraction (D) and the difference in spherical equivalent value (Humphrey - subjective) (D) $(n=160) . A, B, C$, and $D=1,2,3$, and 4 observations, respectively.

$0.25 \mathrm{D}$, and in 439 eyes $(98.0 \%)$ within plus or minus $0.50 \mathrm{D}$.

Concerning cylinder axis, in $223(64 \cdot 8 \%)$ of the 344 eyes with astigmatism, the cylinder axis determined by the autorefractor was within 10 degrees of difference from that measured subjectively, and in 300 eyes $(87 \cdot 2 \%)$ within 20 degrees of difference.

Figure 2 illustrates that there was no obvious relation between the differences in spherical equivalent values measured with the Humphrey autorefractor and subjective refraction, and the values for average spherical equivalent determined by these two methods. Thus, the differences between the values obtained with these two methods did not vary in any systematic manner over the range of measurements. A similar relation was found for the left eye (data not shown) and when comparing the Nidek and subjective refraction for the right and left eye (data not shown).

The differences in spherical equivalent values between the Humphrey and subjective refraction were found to be approximately normally distributed, and thus the limits of agreement between the two methods were calculated. ${ }^{8}$ As shown in Table 1 , the limits of agreement were within plus or minus $0.95 \mathrm{D}$.

Testing the refraction results determined by the Humphrey autorefractor, the mean of the best visual acuity achieved was 0.86 (SD $0.24) \quad(n=444)$, and 1.08 (SD 0.14$)$ $(n=448)$ measured with the best subjective refraction.

AGREEMENT BETWEEN REFRACTION DETERMINED BY THE NIDEK AUTOREFRACTOR AND SUBJECTIVE REFRACTION

Comparing the Nidek autorefractor and subjective refraction, the mean differences in spherical equivalents between these two methods were statistically significant for both eyes (right eye $p=0.0001$, left eye $p=0.0002$ ), although they were less than $-0 \cdot 15 \mathrm{D}$ in both eyes (Table 1).

The spherical equivalent values measured by the autorefractor agreed with the subjective refraction values within plus or minus $0.25 \mathrm{D}$ in 118 of $160(73.8 \%)$ eyes and within plus or minus $0.5 \mathrm{D}$ in $149(93.1 \%)$ eyes. Regarding the sphere component, values recorded in 129 eyes $(80.6 \%)$ by the autorefractor agreed with the subjective refraction within plus or minus $0.25 \mathrm{D}$ and in $153(95.6 \%)$ eyes within plus or minus $0.50 \mathrm{D}$. In 143 eyes $(89.4 \%)$, the cylinder component agreed within plus or minus $0.25 \mathrm{D}$ and in $156(97.5 \%)$ eyes within plus or minus $0.50 \mathrm{D}$.

In 119 eyes with astigmatism, 50 eyes $(42.0 \%)$ had a cylinder axis measured by the autorefractor that agreed with the subjectively measured axis within 10 degrees, and in 92 eyes $(77 \cdot 3 \%)$ within 20 degrees of difference.

The differences in spherical equivalent values between the Nidek and subjective refraction were approximately normally distributed. The limits of agreement between the two methods were calculated, and as shown in Table 1, they were within plus or minus $0.55 \mathrm{D}^{8}$

Testing the refraction results determined by the Nidek autorefractor, the mean of the best visual acuity determined in this way was 0.98 (SD 0.19) $(n=140)$, while it was 1.08 (SD $0 \cdot 16)(n=160)$ determined with the best subjective refraction.

AGREEMENT BETWEEN REFRACTION DETERMINED BY THE HUMPHREY AND THE NIDEK AUTOREFRACTOR

To evaluate the level of precision for both autorefractors, the refractive values obtained by all three methods in 160 eyes were finally compared. Regarding the distribution of refractive errors in these eyes measured by 
the Humphrey autorefractor, the Nidek autorefractor and subjectively, no statistically significant difference $(p=0 \cdot 3828)$ was found using the Kruskal-Wallis test.

Comparing the differences in spherical equivalent values between the two autorefractors and subjective refraction, a distinct divergence was revealed. Thus, evaluating the precision of the autorefractors compared with subjective refraction, $47.5 \%(n=76)$ of the results of the Humphrey autorefractor were within plus or minus $0.25 \mathrm{D}$ limits, compared with $73.8 \% \quad(n=118)$ of the results of the Nidek autorefractor. The McNemar test showed that the difference in precision between these two instruments was statistically. significant $(p<0.001) .10$

\section{Discussion}

As shown in this study, there was a negative difference of means expressed in spherical equivalents between the values determined with the Humphrey and Nidek autorefractors and subjective refraction, indicating that the autorefractors measure more negative and less positive values than subjective refraction. However, this difference was about twice as high for the Humphrey autorefractor than for the Nidek autorefractor. Thus, the systematic bias of autorefraction compared with subjective refraction seems to be greater for the Humphrey autorefractor than the Nidek autorefractor.

Comparing the results reported previously with cycloplegia, Zadnik et al did not find a statistically significant bias when comparing the Canon R-1 autorefractor with subjective refraction. ${ }^{7}$ On the other hand, Wong et al demonstrated the same tendency, as we did, of the Humphrey automatic refractor to measure more negative values compared with clinical refraction. ${ }^{5}$

Nayak et al showed that without cycloplegia, there was an even greater tendency of the Nikon NR-1000 F autorefractor to measure more negative or less positive values than when refracted subjectively. ${ }^{6}$ This may be caused by instrument myopia because the automatic fogging system of some autorefractors probably does not sufficiently control the accommodation. ${ }^{11}$ On the other hand, Wesemann et al found the Humphrey HAR 520 autorefractor to be in good agreement with subjective refraction and the differences in spherical

Table 2 Mean difference in refraction (D) between autorefractors and subjective refraction (autorefractors minus subjective refraction) and $95 \%$ limits of agreement for these differences in four studies. For the two first studies, calculation of limits of agreement has been performed on the basis of the published data. All studies are on cycloplegic eyes

\begin{tabular}{|c|c|c|c|c|}
\hline Authors & $\begin{array}{l}\text { Number } \\
\text { of eyes }\end{array}$ & $\begin{array}{l}S D \\
(D)\end{array}$ & $\begin{array}{l}\text { Mean difference } \\
\text { (D) }\end{array}$ & $\begin{array}{l}95 \% \text { Limits of } \\
\text { agreement (D) }\end{array}$ \\
\hline $\begin{array}{l}\text { Wong et al (1984)5 } \\
\text { (Humphrey HAR) }\end{array}$ & $\begin{array}{l}\text { RE } 40 \\
\text { LE } 40\end{array}$ & $\begin{array}{l}0.54 \\
0.51\end{array}$ & $\begin{array}{l}-0.43 \\
-0.45\end{array}$ & $\begin{array}{l}-1.51 \text { to } 0.65 \\
-1.47 \text { to } 0.57\end{array}$ \\
\hline $\begin{array}{l}\text { Nayak et al (1987) } \\
\text { (Nikon NR-1000F) }\end{array}$ & & 0.25 & -0.02 & -0.52 to 0.48 \\
\hline $\begin{array}{l}\text { Zadnik et al (1992) } \\
\text { (Canon R-1) }\end{array}$ & RE 40 & 0.56 & $0 \cdot 16$ & -0.94 to 1.26 \\
\hline $\begin{array}{l}\text { Present study } \\
\text { (Humphrey 500) } \\
\text { Present study }\end{array}$ & $\begin{array}{l}\text { RE } 224 \\
\text { LE } 224 \\
\text { RE } 80\end{array}$ & $\begin{array}{l}0 \cdot 47 \\
0 \cdot 46 \\
0 \cdot 27\end{array}$ & $\begin{array}{l}-0.23 \\
-0.20 \\
-0.13\end{array}$ & $\begin{array}{l}-1.18 \text { to } 0.71 \\
-1.12 \text { to } 0.72 \\
-0.68 \text { to } 0.41\end{array}$ \\
\hline (Nidek AR-1000) & LE 80 & 0.26 & -0.11 & -0.62 to 0.40 \\
\hline
\end{tabular}

equivalents between the two methods to be equally distributed around zero. ${ }^{2}$

Examining the percentage agreement between the data obtained with autorefractors and by clinical refraction, there was a discrepancy between the two autorefractors used in this study regarding various refractive components. The agreement of the spherical equivalent of the Humphrey autorefractor was about $70 \%$ at the limit of plus or minus $0.5 \mathrm{D}$, but about $90 \%$ at the same level with the Nidek autorefractor. There was a notable difference in percentage agreement concerning the sphere component, whereas percentage agreement of the cylinder component was more alike for these two instruments. Some previous studies made without cycloplegia reported the same tendency of closer agreement between autorefraction and subjective refraction regarding the cylinder component than with the sphere component, while other studies demonstrated the opposite. ${ }^{2-411} 12$ However, in all of these studies, the differences in percentage agreement between sphere and cylindrical components are relatively small.

In cycloplegia, Nayak et al reported a percentage agreement of spherical equivalent and sphere component values between autorefractor (Nikon NR-1000F) and clinical refractive data that closely agree with the values obtained with the Nidek autorefractor, but are better than the values obtained with the Humphrey autorefractor in the present study. ${ }^{6}$ On the other hand, our study demonstrated closer agreement between the cylinder component values. Regarding the cylinder axis, the percentage agreement within 10 degrees between autorefractor measurements and subjective refraction performed without cycloplegia, has been reported to vary between $28 \%$ and $91 \% .^{3}{ }^{12}$ Our study revealed that the Humphrey autorefractor gave a better estimate of the cylinder axis than the Nidek autorefractor.

As mentioned above, the same procedure was followed in all eyes to examine the refractive errors. Some uncertainty remains, however, regarding a possible variation of the level of cycloplegia during the examination period. Being performed first, the measurements with the Humphrey autorefractor would be more subject to influence by incomplete cycloplegia than subsequent measurements using the Nidek autorefractor or subjective refraction. Thus, the possibility of instrument myopia or latent hyperopia would be most pronounced in measurements made using the Humphrey autorefractor in explaining the results obtained.

The results of this study correspond well with the limits of agreement that can be calculated from data published in other studies performed with different autorefractors in cycloplegia (Table 2). Some of the limits of agreement are relatively wide, which has to be taken into account when these instruments are evaluated. As shown in Table 2, the Nidek autorefractor gave relatively narrow limits of agreement, compared with other studies.

In conclusion, the mean differences in 
spherical equivalent values found between Nidek autorefraction and subjective refraction were relatively small, and there was a high percentage agreement between these two methods for both the sphere and cylinder components. In addition, the visual acuity obtained with Nidek autorefraction corresponded well with the visual acuity obtained by subjective refraction. It supports the conclusion that in this study the Nidek autorefractor was found to predict the refractive errors better than the Humphrey autorefractor, with the exception of the cylinder axis.

Furthermore, the results of this study illustrate that autorefraction can be used as a preliminary refractive method. However, in agreement with previous recommendations, the results of our study indicate that autorefraction should be used as a complement to subjective refraction and not as a substitute for it, where cycloplegic readings are concerned. ${ }^{2} 1213$

We thank the participating students at the University of Trondheim. Furthermore, we thank Jarand Rystad, MSc, for data management assistance and John G Taylor, MSc, for assistance with the text.
1 Rassow B, Wesemann W. Automatic infrared refractors 1984. Ophthalmology 1984; 91 (9 suppl): 10-26.

2 Wesemann W, Rassow B. Automated infrared refractors - a comparative study. Am $\mathcal{f}$ Optom Physiol Opt 1987; 64: 627-38.

3 McBrien NA, Millodot $M$. Clinical evaluation of the Canon Autoref R-1. Am f Optom Physiol Opt 1985; 62: 786-92.

4 Sunder Raj P, Villada JR, Lewis AE, Joyce PW, Watson A. Comparative evaluation of the Allergan Humphrey 570 and Canon RK-1 autorefractors. I. Objective autorefraction in normal subjects. Eye 1992; 6: 284-6.

5 Wong EK, Patella M, Pratt M, Myers SW, Gaster RN, Leopold IH. Clinical evaluation of the Humphrey autoLeopold IH. Clinical evaluation of the Humphrey

6 Nayak BK, Ghose S, Singh JP. A comparison of cycloplegic and manifest refractions on the NR-1000F (an objective auto refractometer). Br $\mathcal{f}$ Ophthalmol 1987; 71: 73-5.

7 Zadnik K, Mutti DO, Adams AJ. The repeatability of measurement of the ocular components. Invest Ophthalmol Vis Sci 1992; 33: 2325-33.

8 Bland JM, Altman DG. Statistical methods for assessing agreement between two methods of clinical measurement. Lancet 1986; i: 307-10.

9 Kinge B, Midelfart A. Refractive errors among Norwegian engineering students. Ophthalmic Epidemiology 1994; 1: 5-13.

10 SAS Institute Inc. SAS/STAT guide for personal computers, Version 6 Edition. Cary, NC: SAS Institute Inc, 1987.

11 Ghose S, Nayak BK, Singh JP. Critical evaluation of the NR-1000F autorefractometer. Br $\mathcal{F}$ Ophthalmol 1986; 70: 221-6.

12 Sunder Raj P, Villada JR, Myint K, Lewis A, Akingbehin T. Clinical evaluation of automated refraction in anterior chamber pseudophakia. Br f Ophthalmol 1991; 75: 42-4.

13 Wood ICJ, Papas E, Burghardt D, Hardwick G. A clinical evaluation of the Nidek autorefractor. Ophthalmic Physiol Opt 1984; 4: 169-78. 